\title{
Differential effects of psychopathy and antisocial personality disorder symptoms on cognitive and fear processing in female offenders
}

\author{
Marja E. Anton • Arielle R. Baskin-Sommers • \\ Jennifer E. Vitale • John J. Curtin • Joseph P. Newman
}

Published online: 12 August 2012

(C) Psychonomic Society, Inc. 2012

\begin{abstract}
Psychopathy and antisocial personality disorder (APD) have long been considered important risk factors for criminal behavior and incarceration. However, little is known about the psychobiological underpinnings that give rise to the disinhibited behavior of female offenders. Using an instructed fear-conditioning paradigm and a sample of incarcerated female offenders, we manipulated attentional focus and cognitive load to characterize and differentiate between the dysfunctional cognitive and affective processes associated with these syndromes. We used fear-potentiated startle (FPS) and event-related potentials as measures of affective and cognitive processing, respectively. After controlling for APD symptoms, psychopathic women displayed greater FPS while attending directly to threat-relevant stimuli and displayed less FPS while performing a demanding task that directed attention to threat-irrelevant information. Conversely, controlling for psychopathy, women with high APD symptoms displayed less overall FPS, especially when instructed to focus on threat-relevant stimuli. However, as the demands on cognitive resources increased, they displayed greater FPS. For both psychopathy and APD, analysis of the event-related potentials qualified these findings and further specified the abnormal cognitive processes associated with these two syndromes. Overall, simultaneous analysis of psychopathy and APD revealed distinct patterns of cognitive processing and fear reactivity.
\end{abstract}

M. E. Anton · A. R. Baskin-Sommers $(\bowtie) \cdot J$ J. J. Curtin •

J. P. Newman

Department of Psychology, University of Wisconsin-Madison,

1202 W. Johnson St.,

Madison, WI 53706, USA

e-mail: baskinsommer@wisc.edu

J. E. Vitale

Hampden-Sydney College,

Hampden Sydney, VA, USA
Keywords Female offenders - Attention - Cognition · Fear · Fear-potentiated startle $\cdot$ Event-related potential

Psychopathy and antisocial personality disorder (APD) have long been considered important risk factors for criminal behavior and incarceration. Increasingly, researchers are investigating the significance of these syndromes for female offender populations (Rogstad \& Rogers, 2008; Verona \& Vitale, 2006; Warren \& South, 2006). Among incarcerated women, base rates of psychopathy have been estimated to range from $9 \%-11 \%$ (Loucks, 1995; Neary, 1990; Vitale \& Newman, 2001). For APD, base rates are closer to $21 \%$ (Fazel \& Danesh, 2002). Additionally, Warren and South (2006) reported that approximately $30 \%$ of female offenders with psychopathy also qualify for an APD diagnosis. While most female offenders do not qualify for these categorical diagnoses, women displaying higher levels of psychopathic and APD symptoms are at high risk for diverse conduct problems (e.g., sexual promiscuity, pathological lying, or manipulative behavior), both in prison and upon release. To date, however, little is known about the psychobiological underpinnings that give rise to the disinhibited behavior of female offenders.

Although APD and psychopathy share many features, such as pathological impulsivity, irresponsibility, aggression, and antisocial behavior (Dolan \& Völlm, 2009; Rogstad \& Rogers, 2008; Warren \& South, 2006), they differ in significant ways. Psychopathy, for instance, is distinguished by callous-unemotional traits (e.g., glibness, pathological lying, shallow affect, and lack of empathy) and low levels of anxiety, depression, and general psychopathology (Patrick, 2007). Conversely, women with APD have higher rates of comorbid psychiatric disorders, such as substance abuse, depression, anxiety, and self-injurious behaviors (Mulder, Wells, \& Bushnell, 1994; Rogstad \& Rogers, 2008; Singh \& Waldman, 
2010; Warren \& South, 2006). In terms of criminal behavior, psychopathy in women is associated with higher rates of incarcerations and a greater likelihood of committing both violent and nonviolent crimes (Louth, Hare, \& Linden, 1998; Strachan, 1995; Vitale, Smith, Brinkley, \& Newman, 2002). Women with APD are more likely to display generally impulsive and irresponsible behavior (e.g., chronic unemployment, high rates of marital separation, or dependence on welfare), though this behavior is not necessarily criminal (Mulder et al., 1994).

The distinction between psychopathy and APD is further clarified by factor-analytic studies based on the Psychopathy Checklist-Revised (PCL-R; Hare, 2003), which have identified two sets of items that are differentially associated with APD. Factor 1 reflects the interpersonal (charm, grandiosity, and deceitfulness/conning) and affective (lack of remorse, empathy, and emotional depth) features of psychopathy. Alternatively, Factor 2 describes the impulsive and chronic antisocial tendencies associated with psychopathy (Kennealy, Hicks, \& Patrick, 2007). In comparison to those with APD, female offenders with high levels of Factor 1 constitute a recidivistic offender subtype (Salekin, Rogers, \& Sewell, 1997). Factor 2, though, is primarily related to early behavioral problems, sexual promiscuity, and adult antisocial behavior, which significantly overlap with APD symptoms (Patrick, Hicks, Krueger, \& Lang, 2005; Salekin et al., 1997; Vaidyanathan, Hall, Patrick, \& Bernat, 2011; Warren et al., 2003). The differential traits associated with psychopathy and APD yield important distinctions in their behavior patterns, and potentially in their underlying psychobiological processes.

Beyond characterizing and distinguishing the symptoms and personality correlates of psychopathy and APD, it is important to clarify the extent to which these syndromes reflect similar or distinct psychological dysfunction in female offenders. While research on psychopathy and APD in males has elucidated important attentional, cognitive, and emotion processing abnormalities in these syndromes (BaskinSommers \& Newman, in press; Blair, 2003; Glenn \& Yang, 2012; Patrick, 2007), little has been done to characterize these processes in female offenders. Thus, relative to the literature on males, the processes contributing to disinhibited behavior in female offenders are less well specified, and it is unclear whether psychopathy and APD reflect similar or distinct predispositions to disinhibited behavior.

Existing research on psychopathy in women has generated equivocal findings, but it has provided evidence that deficient emotional reactivity and abnormalities in selective attention may contribute to disinhibited behavior. Sutton, Vitale, and Newman (2002) found that psychopathic women displayed significantly less emotion-modulated startle (i.e., startle potentiation) than did nonpsychopathic women while viewing unpleasant versus neutral pictures. However, this effect was observed only when startle probes were delivered soon after picture onset (i.e., at 2 -s but not at 4.5-s probe times), indicating that this emotion deficit was conditional rather than fundamental, in an absolute sense. Moreover, investigations of passive avoidance learning and emotion facilitation by affective word stimuli- two emotion-based deficits of particular relevance for psychopathy — did not find these affective deficits in psychopathic women (Vitale, MacCoon, \& Newman, 2011; Vitale \& Newman, 2001). Thus, existing research does not indicate the presence of a reliable emotional deficit in psychopathic female offenders, though such deficits may be revealed under specific circumstances (e.g., Sutton et al., 2002).

One explanation for the inconsistent nature of psychopathyrelated emotion deficits may relate to an abnormality in early selective attention. Using modified Stroop tasks, Vitale, Brinkley, Hiatt, and Newman (2007) demonstrated that female psychopathic offenders over-focused on goal-relevant stimuli and were significantly less influenced by conflicting contextual information than were nonpsychopathic offenders. More generally, psychopathic individuals appear oblivious to potentially meaningful peripheral information because they fail to reallocate attention while engaged in goal-directed behavior (MacCoon, Wallace, \& Newman, 2004; Patterson \& Newman, 1993). Consequently, it may be that an abnormality in early selective attention acts to limit attention to dominant or prepotent goal-relevant stimuli. This type of deficit may play a crucial role in moderating the expression of emotion (i.e., fear) deficits in female psychopathic offenders; however, this prediction has yet to be rigorously examined in a female sample.

With regard to APD, women with APD are thought to lack inhibitory control over affective and behavioral reactions and, thus, to be prone to disinhibited reactions in the presence of salient emotion cues (Litt, Hien, \& Levin, 2003; Sylvers, Brennan, Lilienfeld, \& Alden, 2010). Consistent with this view, Zlotnick (1999) found that incarcerated women with APD displayed significantly more emotion dysregulation than did controls, especially poor anger modulation. Similarly, Lorenz and Newman (2002) found that women with APD exhibited more emotion facilitation on a lexical decision task than did female offenders without APD and, furthermore, that higher levels of emotion facilitation in women with APD were associated with higher rates of violent crime. While it is not possible to draw causal inferences from correlational data, these findings suggest a potential link between hyperreactivity to emotion information and their disinhibited behavior. Also of potential relevance, Litt, Hien, and Levin (2003) found that women with adult antisocial behavior had a significantly reduced capacity for affect tolerance and affect expression relative to women without adult antisocial behavior. Overall, such evidence suggests that women with APD display hyperarousal or reactivity in response to affective information (see also 
Ogle, Maier-Katkin, \& Bernard, 1995; Zahn-Waxler, Shirtcliff, \& Marceau, 2008).

A straight-forward explanation for the emotional hyperreactivity of women with APD is that the intensity of their emotion reactions is so powerful and pervasive that it directly gives rise to exaggerated emotion-related responses. However, the executive-functioning perspective suggests that a deficit in regulating high-order processes (e.g., working memory and cognitive control) may indirectly amplify emotional reactivity because individuals who do not have the capacity to maintain control are prone to overreact to emotional information (Beauchaine, Klein, Crowell, Derbidge, \& Gatzke-Kopp, 2009; Morgan \& Lilienfeld, 2000). Such findings are consistent with the possibility that antisocial behavior is associated with differences in executive control (e.g., Morgan \& Lilienfeld, 2000; Ogilvie, Stewart, Chan, \& Shum, 2011) and that women with antisocial tendencies are less adept at modulating affective arousal (see Sargeant, Daughters, Curtin, Schuster, \& Lejuez, 2011).

Overall, the literature evaluating the psychobiological mechanisms contributing to psychopathy and APD in female offenders is, as yet, quite limited. Consistent with the phenotypic distinctions between these syndromes, the existing literature suggests that the cognitive and affective signatures of women with psychopathy and APD are in fact distinct. However, to our knowledge, no study has compared the psychobiological mechanisms in women with psychopathy versus APD in order to examine the extent to which they are the same or different. As such, not only was the present study designed to investigate the cognitive and affective (i.e., fear reactivity) dysfunctions associated with psychopathy and APD in female offenders, but also to contrast the respective syndrome-related processes.

More specifically, we assessed fear-potentiated startle (FPS) and event-related potentials (ERPs) in a sample of incarcerated female offenders under experimental conditions that (1) focused attention directly on threat-relevant information (i.e., a color predicting electric shocks), (2) established an alternative attentional focus that placed minimal demands on working memory (i.e., low load), and (3) established an alternative attentional focus that placed relatively high demands on working memory (i.e., high load). FPS was used as a psychophysiological measure of fear reactivity (Davis, Falls, Campeau, \& Kim, 1993) and has been found to be sensitive to changes in cognitive demands (i.e., reactivity decreases under conditions of high cognitive load; Pessoa, McKenna, Gutierrez, \& Ungerleider, 2002). Additionally, we used ERPs to probe the contributions of cognitive processes at different points in the processing stream. Briefly, ERPs at an early stage, such as P1, generally reflect early selective attention (i.e., filtering), whereas later ERPs, like P3, reflect capacity-limited cognitive processes such as categorization of information and response selection (e.g., Luck, Woodman, \& Vogel, 2000). Given this paradigm and the proposed psychobiological mechanisms contributing to psychopathy and APD, respectively, we investigated hypotheses with the aim of characterizing and disambiguating the cognitive and affective processes associated with these syndromes.

Psychopathy A key question for understanding the psychobiology of psychopathy in women is the extent to which attention abnormalities contribute to the information-processing (i.e., affective information) deficits of these individuals. If attentional focus does not moderate the fear reactivity of psychopathic offenders, then high psychopathy scores will be associated with either an absolute deficit in FPS (i.e., across all three conditions) or normal fear reactivity across conditions. Conversely, if the hypothesized attention abnormality moderates fear deficits in psychopathy (Newman \& Baskin-Sommers, 2011), we should observe a significant Psychopathy $\times$ Condition interaction, with psychopathic participants displaying greater FPS in the threatfocused condition (i.e., when threat cues are the explicit focus of attention) than in the two alternative-focus conditions (i.e., when threat cues are peripheral to an alternative goal). Moreover, the ERP data will assist in specifying the time course of the predicted attentional abnormalities. If, as proposed by Newman and colleagues (2011), the attention dysfunction operating in psychopathy is related primarily to dysfunction at an early stage of selective attention, we would expect to see psychopathyrelated differences starting at an early ERP (e.g., P1). More specifically, if psychopathic female offenders have a tendency to establish a rigid focus on goal-relevant stimuli and to filter out distractors, this type of processing should result in larger P1 responses, especially during the alternative-focus conditions (i.e., when there is a need to screen out the distracting threat information in order to respond to information about the letter).

Antisocial personality disorder There are alternative hypotheses regarding the mechanisms contributing to disinhibition in APD. One possibility is that women with APD are characterized by a general hyperreactivity to threat cues, whereas another possibility is that their hyperreactivity is moderated by a deficit in capacity-limited processes. If the affective dysregulation associated with APD reflects a more general hyperreactivity to threat cues, it follows that APD would be associated with greater FPS across all three conditions. Conversely, if their affective dysregulation is moderated by demands on cognitive capacity, APD-related abnormalities in FPS would vary as a function of cognitive load. More specifically, their reaction to peripheral threat cues (i.e., FPS) should be larger (i.e., disinhibited) under conditions involving high versus low cognitive/working memory load. Moreover, in as much as APD is associated with a deficit in capacity-related functions, this deficit should be especially apparent in ERP responses that tap 
capacity-dependent processes (e.g., P3). That is, under the conditions that establish a need to focus on goal-directed information and to screen out distractors, $\mathrm{P} 3$ responses may be diminished and provide differential support for a deficit in cognitive capacity that moderates affective reactivity in high-APD female offenders.

Finally, in addition to evaluating these process-level dysfunctions in psychopathy and APD, we also investigated the extent to which psychopathy-related effects pertain to the unique variance associated with PCL-R Factor 1 (interpersonal and affective features) and Factor 2 (impulsive and antisocial features). In light of previous research linking psychopathyrelated deficits to Factor 1 traits (see Kennealy et al., 2007), it follows that the results for Factor 1 traits may correspond to the findings for psychopathy total score. Conversely, given the strong association between PCL-R Factor 2 and APD, it follows that the PCL-R Factor 2 effects may resemble the APD effects (Verona \& Vitale, 2006).

\section{Method}

\section{Participants}

The participants were 84 Caucasian female offenders from a minimum-security correctional center in southern Wisconsin. Because there is little evidence that psychopathy-related laboratory correlates generalize across race and gender, our laboratory disaggregates these samples and reports sample-specific effects in separate studies (Baskin-Sommers, Newman, Sathasivam, \& Curtin, 2011; Vitale et al., 2011). All participants were between the ages of 18 and 45, because psychopathy and impulsive behavior have been found to change with advancing age (Hare, Harpur, \& Hakstian, 1990). Additionally, a prescreen of institutional files was used to exclude individuals who had performed below the fourth-grade level on a standardized measure of reading or math achievement, who scored below 70 on a brief measure of IQ (i.e., the Shipley Institutes of Living Scale; Zachary, 1986), or who had diagnoses of schizophrenia, bipolar disorder, or psychosis not otherwise specified. The intelligence cutoff and exclusion of major psychopathology were used primarily to reduce the contributions of these extraneous influences on the interview and psychophysiological assessments.

\section{Assessment measures}

Psychopathy checklist-revised (Hare, 2003) PCL-R ratings were completed using information from prison files and a semistructured interview that lasted approximately $60 \mathrm{~min}$. The PCL-R contains 20 items that are rated 0,1 , or 2 , reflecting the degree to which a characteristic is present: significantly (2), moderately (1), or not at all (0). In the present sample, scores on this measure ranged from 6.3 to 36 (total possible range is 0 to 40$)$, with a mean of $21.50(S D=6.54)$. Interrater reliability (intraclass correlation) for PCL-R total score, based on 21 dual ratings, was .99 .

Early work with the PCL-R has also revealed a replicable two-factor structure (Hare et al., 1990), with Factor 1 items assessing interpersonal-affective characteristics (e.g., glibness or callousness) and Factor 2 items relating to impulsive-antisocial behavior (e.g., irresponsible behavior or criminality). In the present sample, scores on PCL-R Factor 1 ranged from 0 to 14 (total possible range is $0-16)$, with a mean of $7.74(S D=$ 3.31), and scores on PCL-R Factor 2 ranged from 1 to 20 (total possible range is $0-20)$, with a mean of $11.70(S D=3.81)$. The interrater reliabilities for PCL-R factor scores were .94 and .97 for Factors 1 and 2, respectively.

Antisocial personality disorder symptoms (APD) Participants were also assessed for APD symptoms during the same interview and file review used in the PCL-R assessment, using specific questions and information concerning a variety of antisocial behaviors (e.g., burglary, physical cruelty to animals, and vandalism). Following the Diagnostic Statistical Manual-IV (DSM-IV) criteria, APD symptom scores were calculated by summing the number of childhood (15 years and younger) conduct disorder symptoms plus the adult criteria met for each participant. The mean number of APD symptoms in this sample was $6.14(S D=$ 2.89), and scores on this measure (i.e., numbers of symptoms met) ranged from 1 to 14 (total possible range 0 to 22 for child/adult symptoms). Cronbach's alpha for the APD total score was .73. In this sample, the interrater reliability for this measure was .95 .

\section{Procedure}

Individuals meeting the inclusion criteria (see the Participants section) were invited to participate in an ongoing study. All participants provided written informed consent according to procedures approved by the University of WisconsinMadison Human Subjects Committee. On the first day of the study, inmates were called to a private office and completed a semistructured life history interview with one of five experienced interviewers. Following the interview, the examiner reviewed the institutional file in order to corroborate information provided during the interview and to record official encounters with the criminal justice system. The combination of interview and file information was used to rate psychopathy and APD and to quantify the number and types of criminal charges. Participants were paid $\$ 8$ for completing the initial assessment.

On a second day, the participants returned for an experimental session. Upon arrival, participants were asked to wash their faces, and one of the three female experimenters 
prepared the face and scalp for the electrode application. Following this setup, the experimenter administered the shock sensitivity procedure (see the Shock Sensitivity section below). Following this procedure, participants received verbal instructions for the instructed fear-conditioning task. These instructions included the information that, in all conditions, electric shocks might be administered on some trials following red letters (threat), but that no shocks would follow green letters (no threat). Finally, participants performed the instructed fear-conditioning task, which lasted approximately $30 \mathrm{~min}$. Participants earned $\$ 25$ for their completion of the task.

\section{Shock sensitivity}

In this procedure, the intensity of shocks is calibrated to each participant's subjective shock sensitivity. More specifically, participants are administered a series of electric shocks of increasing intensity to the fingers of their nondominant hand. Participants report two intensity anchors: The first is the intensity that they consider uncomfortable, and the second is the maximum intensity level that they can tolerate. The series is terminated when they reach their maximum intensity level. The absolute range of shock current was 0-7 mA. During the main task, participants received shocks calibrated at the midpoint between their first and second intensity anchors.

The purpose of this procedure was to control for individual differences in level of shock sensitivity during the experimental task. Of note, there was no relationship between psychopathy total scores $(p=.27)$, the psychopathy factors (Factor 1, $p=.15$; Factor $2, p=.74$ ), or APD symptoms $(p=.60)$ and the maximum intensity level of shock intensity reported.

\section{Instructed fear-conditioning task}

The instructed fear-conditioning task was identical to the one described by Newman, Curtin, Bertsch, and Baskin-Sommers (2010). The task involved three conditions, consisting of 100 trials per condition. Each trial involved the presentation of an upper- or lowercase letter appearing in a red or green font for $400 \mathrm{~ms}$, followed by a variable (i.e., 2- to 2.8-s) intertrial interval. Because the letters were not preceded by a warning stimulus or followed by response feedback, these appeared as a seamless series of 100 letters.

In all three conditions, participants pressed one of two buttons according to the instructions associated with each condition (described below). In all three conditions, electric shocks were presented following $20 \%$ of the red letters and $0 \%$ of the green letters to establish the red and green letters as threat and no-threat stimuli, respectively. Shocks were administered for $200 \mathrm{~ms}$ to adjacent fingers on the participant's left hand at 1,400-ms post-stimulus-onset. A total of 48 startle-eliciting noise probes $(50-\mathrm{ms}, 102-\mathrm{dB}$ white noise bursts with near instantaneous rise time) were presented $1,400 \mathrm{~ms}$ after stimulus onset. The noise probes were equally distributed across threat/no-threat trials in all three conditions, so that each participant experienced 16 noise probes: eight red (threat) and eight green (no threat) per condition. The average time between noise probes was $28.8 \mathrm{~s}$, with a minimum of $13.7 \mathrm{~s}$. Additionally, probes never occurred in the same trial as a shock administration.

The three conditions were designed to manipulate the focus of attention and to sequentially increase the demands on cognitive load. In the threat-focused condition (TF), participants were instructed to attend to the color of the letter cue and to press one of two buttons using their right hand, according to whether the letters indicated threat (red) or no threat (green). To further enhance the threat focus manipulation and to increase task motivation, participants were informed that speed and accuracy would influence the amount of shocks that they received. In fact, however, the number of shocks received was unrelated to behavioral performance. In the alternative-focus/low-load condition (AF/LL), participants had to determine whether the letter stimuli were upper- or lowercase and to press the appropriate button with their right hand. In the alternative-focus/ high-load condition (AF/HL), participants performed a two-back task in which they had to attend to each letter in the series and press one of the two buttons to indicate whether the current letter matched the letter presented two trials back in the series. In both AF conditions, the letter color (threat information) was peripheral to the primary task and was not necessary to perform the task well (i.e., to identify the case of the letter or perform the two-back task). Moreover, to further enhance task motivation in the $\mathrm{AF}$ conditions, participants were informed that speed and accuracy would influence the amount of reward that they received.

Physiological recording and data reduction

The stimulus presentation and data collection were controlled by a PC-based DMDX script (Forster \& Forster, 2003) and Neuroscan Synamps amplifiers and acquisition software (Compumedics, North Carolina). Offline data processing was conducted with the PhysBox plugin (Curtin, 2011) within the EEGLab toolbox (Delorme \& Makeig, 2004) in MATLAB.

Startle response measurement Startle eyeblink electromyographic activity was sampled at $2,000 \mathrm{~Hz}$ with a bandpass filter $(30-500 \mathrm{~Hz}, 24-\mathrm{dB} /$ octave roll-off) from electrodes placed on the orbicularis oculi muscle under the right eye. 
Offline processing included epoching (50-250 ms surrounding noise probe), rectification and smoothing $(30-\mathrm{Hz}$ lowpass filter following rectification), and baseline correction. Startle blink magnitude was scored as the peak response between 20 and $120 \mathrm{~ms}$ post-probe-onset. Fear response to threat cues was indexed by FPS, calculated as the difference in blink response magnitude to probes following red (threat) as compared with green (no-threat) trials in each of the three task conditions.

Event-related potentials $\mathrm{EEG}$ was recorded from $\mathrm{Ag}-\mathrm{AgCl}$ electrodes mounted in an elastic cap (Electro Cap International) at the standard midline positions $(\mathrm{Fz}, \mathrm{FCz}, \mathrm{Cz}$, and $\mathrm{Pz}$ ) and at the left and right occipital positions (O1 and $\mathrm{O} 2$ ) referenced to the left mastoid. Vertical eye movement was measured with additional electrodes placed above and below the left eye. The electrode impedance for all channels was kept below $10 \mathrm{k} \Omega$. Electroencephalography was recorded at a $2500-\mathrm{Hz}$ sampling rate.

Offline processing included re-referencing to average mastoids, low-pass filtering (2nd-order, 30-Hz Butterworth low-pass filter), epoching (-500 to 1,390-ms epochs ending prior to the onset of startle/shock), baseline correction, and artifact rejection (trials with voltages exceeding $\pm 75 \mu \mathrm{V}$ were rejected). ERPs were averaged separately for all correct trials within each cue type (threat vs. no threat) and task condition (TF, AF/LL, and AF/HL). A minimum of at least 30 trials in each condition was established and an average of 42.6 (out of 50) trials remained in each category after artifact rejection. As a result of the artifact rejection, ERP data were available for only 74 of the 84 participants described above. Inspection of the grand-average ERP waveforms for all participants across all conditions (see Fig. 1) revealed positive potentials that peaked at $82 \mathrm{~ms}(\mathrm{P} 1)$ and $378 \mathrm{~ms}$ (P3) post-stimulus-onset and were maximal at the occipital (average of $\mathrm{O} 1$ and $\mathrm{O} 2$ ) and Pz sites, respectively. The magnitude of $\mathrm{P} 1$ was measured as the mean amplitude in the window of 57-107 ms poststimulus, and the P3 was measured as the mean amplitude in the window of 353$403 \mathrm{~ms}$ post-stimulus-onset.

\section{Data analysis}

The overall goal of our analyses was to distinguish between the cognitive and affective processes operating in psychopathy and APD in female offenders. Toward this end, we used analyses that enabled us to parse the independent (i.e., unique) effects of each syndrome. More specifically, for each measure (e.g., FPS and ERP), we entered continuous measures of psychopathy and APD symptoms into the same general linear model (GLM) to extract the unique effects of these syndromes on the cognitive-affective indices. Following Newman et al. (2010), our primary statistical focus was on two orthogonal
Helmert contrasts that compared the TF condition to the two $\mathrm{AF}$ conditions (attentional focus contrast) and the $\mathrm{AF} /$ LL condition to $\mathrm{AF} / \mathrm{HL}$ condition (cognitive load contrast). These two contrasts enabled us to specify the impacts of attentional focus (i.e., directly focusing on threat vs. focusing on a threat-irrelevant goal) and cognitive load (i.e., low vs. high working memory load), respectively, on these psychophysiological measures.

First, with FPS we used a GLM with Condition (TF, AF/LL, or $\mathrm{AF} / \mathrm{HL}$ ) as a within-subjects categorical factor and PCL-R Psychopathy and APD Symptom Scores (z-scored), entered simultaneously, as between-subjects quantitative factors. Second, we used separate GLMs to analyze P1 and P3 responses. These models included condition (TF, AF/LL, or $\mathrm{AF} / \mathrm{HL}$ ) and cue type (threat vs. no threat) as within-subjects categorical variables, and PCL-R and APD symptom scores, entered simultaneously, as between-subjects quantitative variables. ${ }^{1}$ Again, the inclusion of psychopathy and APD in the same model provided an opportunity to compare the unique effects of these syndromes on ERP responses. As noted in the introduction, for all measures we also reanalyzed the data using the PCL-R factor scores in place of the PCL-R total score (i.e., PCL-R Factor 1 and Factor 2, in the model with APD).

\section{Results}

Descriptive statistics and correlations

Table 1 reports the means and standard deviations for the PCL-R and APD symptom scores and for select behavioral correlates, as well as the correlations among these measures. Though the main analyses quantified psychopathy and APD symptoms continuously, some work with these syndromes has implemented cut scores to classify inmates as psychopathic or having antisocial personality disorder. An exact cut score on the PCL-R has not been established for women, but a range of

\footnotetext{
${ }^{1}$ Although this experiment was designed to examine the effects of attentional and high-order processes on FPS and ERPs, participants were required to make a response on every trial in all three conditions of this experiment. Response times and accuracy were analyzed within a separate general linear model (GLM) with Condition (TF vs. AF/LL vs. AF/HL) and Cue Type (threat vs. safety) as within-subjects, categorical factors and Psychopathy Score (total or factors, standardized) and APD (standardized) as between-subjects, quantitative factors. Consistent with the increasing cognitive demands across conditions, a significant main effect of condition was observed, $F(2,158)=$ $457.46, p<.001$, with response times increasing from the TF $(M=$ $438.6 \mathrm{~ms})$ to $\mathrm{AF} / \mathrm{LL}(M=545.0 \mathrm{~ms})$ to $\mathrm{AF} / \mathrm{HL}(M=875.8 \mathrm{~ms})$ conditions. Additionally, accuracy significantly decreased as cognitive demands increased, $F(2,160)=82.34, p<.001(\mathrm{TF}, M=97.2 \%$; AF/ $\mathrm{LL}, M=93.6 \%$; AF/HL, $M=76.6 \%$ ). However, neither response time nor accuracy was associated with cue type (response time, $p=.76$; accuracy, $p=.23$ ). Additionally, we found no significant psychopathy, $\mathrm{APD}$, or factor-related effects on response times or accuracy.
} 

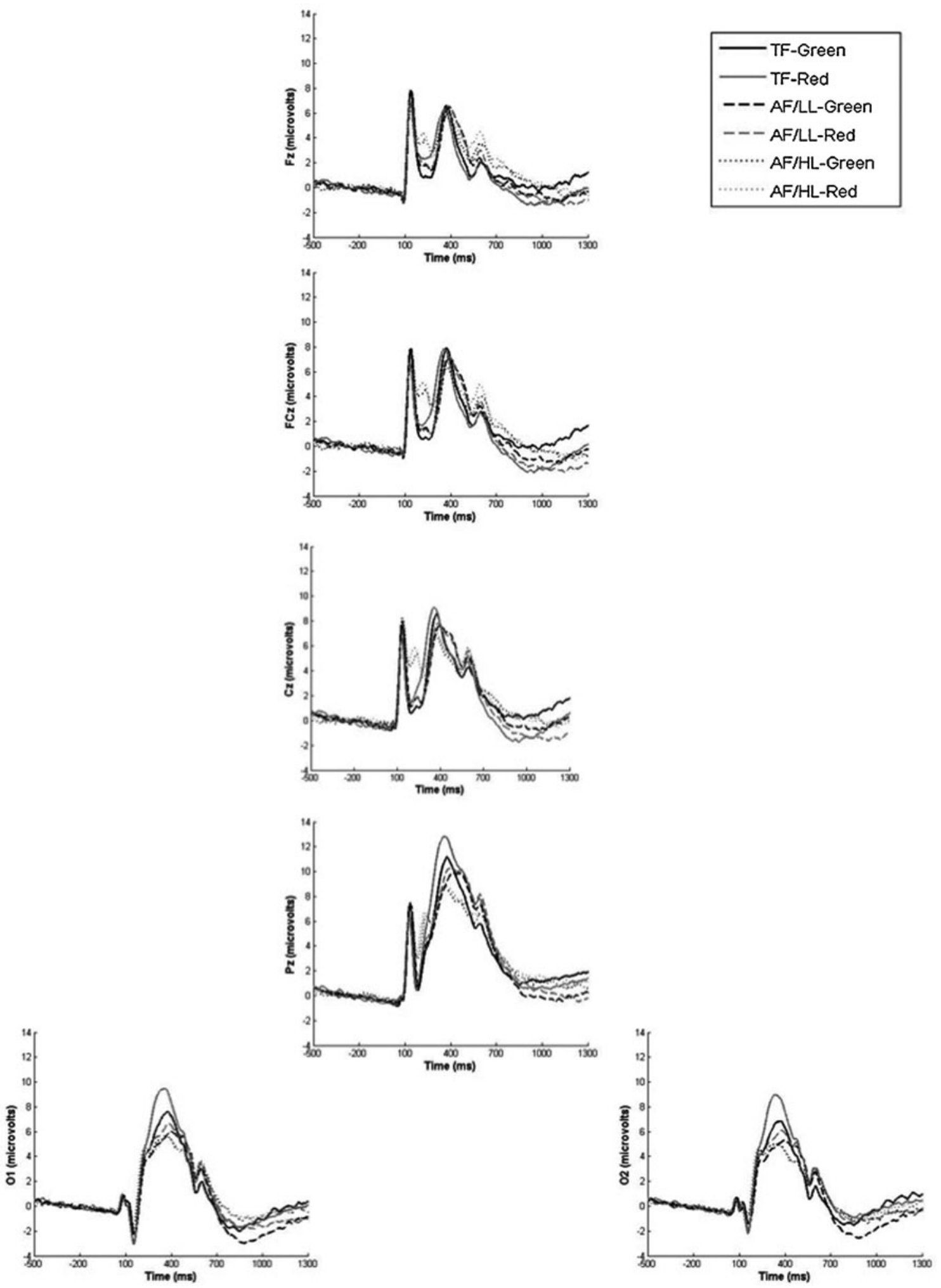

Fig. 1 Stimulus-locked average event-related potential waveforms in the three task conditions for all participants. A digital low-pass filter was applied offline before plotting the waveforms shown here

24-26 has been used previously (Sturek, Loper, \& Warren, 2008; Vitale et al., 2007; Vitale et al., 2011; Warren \& South, 2006). Using a cut score of 25 on the PCL-R and the DSM-IV categorical cut for APD, $8.3 \%$ of the present sample had psychopathy only, $11.9 \%$ had APD only, and $23.8 \%$ had APD and psychopathy. Notably, in the present sample, the rates 
Table 1 Descriptive and bivariate information

$\begin{array}{llll}\text { Mean } & \text { Std. Dev. } & \text { Correlation } & \text { Correlation } \\ \end{array}$

(w/ PCL-R Total) $\quad$ (w/ APD)

Demographic

\begin{tabular}{|c|c|c|c|c|c|}
\hline Age & 32.80 & 6.80 & $19.00-45.00$ & -.18 & -.18 \\
\hline Education (\# years) & 10.79 & 1.24 & $8.00-12.00$ & -.03 & -.12 \\
\hline Estimate of intelligence & 97.22 & 9.66 & $72.81-119.25$ & -.15 & -.07 \\
\hline \multicolumn{6}{|l|}{ Personality Assessment } \\
\hline PCL-R total score & 21.50 & 6.54 & $6.30-36.00$ & - & $.76^{*}$ \\
\hline APD symptoms & 6.14 & 2.89 & $0.00-14.00$ & $.76^{*}$ & - \\
\hline PCL-R Factor 1 & 7.74 & 3.31 & $0.00-14.00$ & $.78^{*}$ & $.47^{*}$ \\
\hline PCL-R Factor 2 & 11.70 & 3.81 & $1.00-20.00$ & $.86^{*}$ & $.73^{*}$ \\
\hline \multicolumn{6}{|l|}{ Adult Crime Information } \\
\hline Total \# of crimes & 16.35 & 89.29 & $0.00-82.00$ & $.31^{*}$ & $.30^{*}$ \\
\hline$\#$ of types of crimes & 4.39 & 12.66 & $0.00-8.00$ & $.51^{*}$ & $.39^{*}$ \\
\hline \# of nonviolent crimes & 15.27 & 12.62 & $0.00-82.00$ & $.29^{*}$ & $.30^{*}$ \\
\hline \# of violent crimes & 1.07 & 3.70 & $0.00-33.00$ & .09 & -.01 \\
\hline
\end{tabular}

PCL-R, Psychopathy Checklist-Revised; APD, antisocial personality disorder. None of the primary analyses were altered when a measure of IQ (estimated WAIS score, based on Shipley measure), age, or years of education were entered into the models. Flagged $\left(^{*}\right)$ cells indicate significant $(p<$ $.05)$ correlations $(r)$ involving PCL-R or APD scores

of psychopathy and APD are in line with the reported base rates in a variety of samples (see Rogstad \& Rogers, 2008; Vitale et al., 2007; Vitale et al., 2002; Warren \& South, 2006).

Additionally, Tables 2 and 3 provide descriptive statistics for the dependent psychophysiological measures used in this study. Given that all of the analyses used continuous between-subject factors, these tables present point estimates at one standard deviation above and below the sample mean to represent high and low estimates on psychopathy and APD. Significant partial and zero-order correlations are also flagged in these tables.
Fear-potentiated startle analyses

The main effect of condition was significant $[F(1,83)=$ $\left.59.26, p .01, \eta_{p}^{2}=.42\right]$. Follow-up orthogonal contrasts indicated that FPS was significantly larger in the TF condition than in the AF conditions (attentional focus contrast; $p<.01$ ) and significantly larger in the AF/LL condition than in the $\mathrm{AF} / \mathrm{HL}$ condition (cognitive load contrast; $p<.01$ ). This pattern of condition effects is consistent with the existing literature, which suggests that as cognitive load increases,

Table 2 General linear model point estimates (and standard errors) for startle response associated with no-threat and threat cues as a function of Psychopathy Checklist-Revised (PCL-R) psychopathy total score, antisocial personality disorder (APD), and task condition

\begin{tabular}{|c|c|c|c|c|}
\hline & Low PCL-R & High PCL-R & Low APD & High APD \\
\hline \multicolumn{5}{|c|}{ Threat Focused } \\
\hline No threat & $99.43(15.75)$ & $94.84(15.75)$ & $85.24(15.75)$ & $109.03(15.75)$ \\
\hline Threat & $117.90(16.63)$ & $134.13(16.63)$ & $130.43(16.63)$ & $121.59(16.63)$ \\
\hline FPS & $18.46^{\dagger}(6.08)$ & $39.29^{\dagger}(6.08)$ & $45.19^{* \dagger}(6.08)$ & $12.55^{* \dagger}(6.08)$ \\
\hline \multicolumn{5}{|c|}{ Alternative Focus/Low Load } \\
\hline No threat & $104.63(16.43)$ & $108.60(16.43)$ & $102.51(16.43)$ & $110.73(16.43)$ \\
\hline Threat & $114.46(16.69)$ & $125.47(16.70)$ & $124.58(16.70)$ & $115.34(16.69)$ \\
\hline FPS & $9.82(5.72)$ & $16.87(5.72)$ & $22.08(5.72)$ & $4.61(5.72)$ \\
\hline \multicolumn{5}{|c|}{ Alternative Focus/High Load } \\
\hline No threat & $110.78(16.00)$ & $115.49(16.00)$ & $106.00(16.00)$ & $120.27(16.00)$ \\
\hline Threat & $113.28(16.40)$ & $110.56(16.40)$ & $106.13(16.40)$ & $117.71(16.40)$ \\
\hline FPS & $2.50^{*}(4.06)$ & $-4.94^{*}(4.06)$ & $.13(4.06)$ & $-2.57(4.06)$ \\
\hline
\end{tabular}

Point estimates are provided for low psychopathy and APD (1 standard deviation below sample mean) and high psychopathy and APD (1 standard deviation above sample mean). Flagged cells indicate significant $(p<.05)$ zero-order $\left(^{*}\right)$ and partial $\left({ }^{\dagger}\right)$ correlations involving PCL-R or APD scores 
the affective response (i.e., FPS) decreases (Newman et al., 2010; Pessoa et al., 2002).

Unique effects of psychopathy total score and APD symptoms The main effect of psychopathy, controlling for APD, was not significant $(p=.24)$, indicating that psychopathy total scores were not consistently related to FPS across conditions.

The orthogonal contrasts indicated that the unique effect of psychopathy interacted with the attentional focus contrast, $F(1,81)=4.19, p=.04, \eta_{p}^{2}=.049$. As can be seen in Fig. 2a, psychopathy was positively and significantly associated with FPS in the TF condition $(B=10.81, p=.04)$, but negatively and nonsignificantly associated with FPS in the AF conditions $(B=-0.100, p=.98)$.

The main effect of APD symptoms, controlling for psychopathy, was statistically significant, $F(1,81)=8.09, p=.01, \eta_{\bar{p}}^{2}=$ .09. Across conditions, female offenders with high APD symptoms displayed reduced FPS. However, this main effect was further qualified by a significant interaction, which indicated that the unique effect of APD interacted with the attentional focus contrast, $F(1,81)=4.82, p=.03$, $\eta_{p}^{2}=.056$. As is shown in Fig. $2 \mathrm{~b}$, APD was significantly and negatively associated with FPS in the TF condition $(B=-17.35, p<.01)$ and nonsignificantly, but still negatively, associated with FPS in the two AF conditions $(B=-5.36, p=.12)$.

Unique effects of psychopathy factor scores and APD symptoms When the PCL-R factor scores were substituted for the PCL-R total score (i.e., to examine the unique variance associated with each factor; see Patrick, 2007), no effects were associated with PCL-R Factor 1 (all $p$ values $>$ .72). For PCL-R Factor 2, both the attentional focus and cognitive load contrasts reached trend-level significance, $\left[F(1,80)=3.32, p=.07, \eta_{p}^{2}=.04\right]$ and $[F(1,80)=3.43$, $\left.p=.07, \eta_{p}^{2}=.041\right]$, respectively. Furthermore, examination of the simple effects to clarify these interactions revealed a significant positive association between PCL-R Factor 2 and FPS in the TF condition ( $B=12.52, p=.01)$, but a nonsignificant association between PCL-R Factor 2 and FPS in the AF conditions $(B=3.271, p=.30)$. Finally, PCL-R Factor 2 was associated with weaker FPS in the AF/HL condition $(B=$ $-1.536, p=.65)$ than in the AF/LL condition $(B=8.078, p=$ $.09)$. The unique effects of APD were essentially equivalent to those found in the analysis employing PCL-R total scores.

Early attention (P1) analyses

Analysis of the P1 response revealed no main effects of cue type $(p=.21)$ or condition $(p=.56)$. Importantly, none of the interaction effects (with APD or psychopathy) involving cue type were significant. Thus, we collapsed across cue types

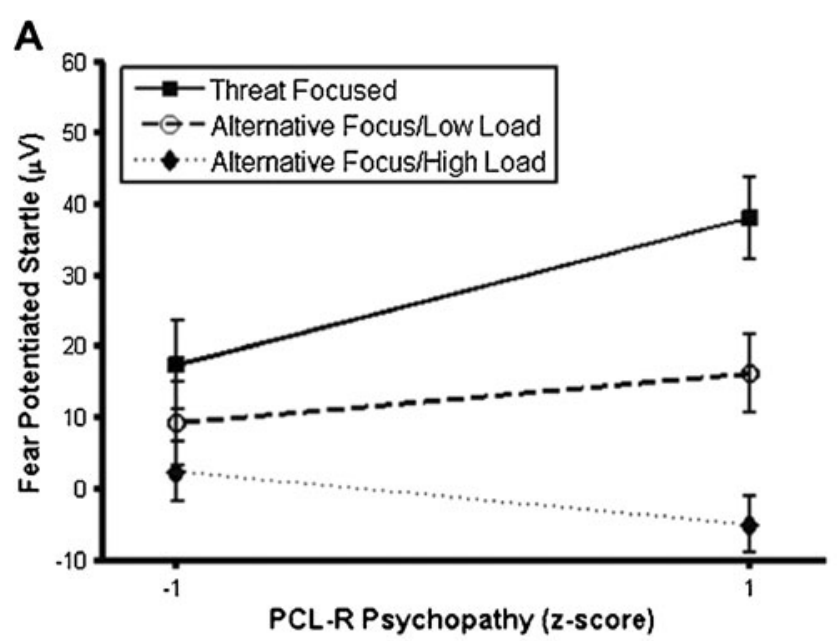

B

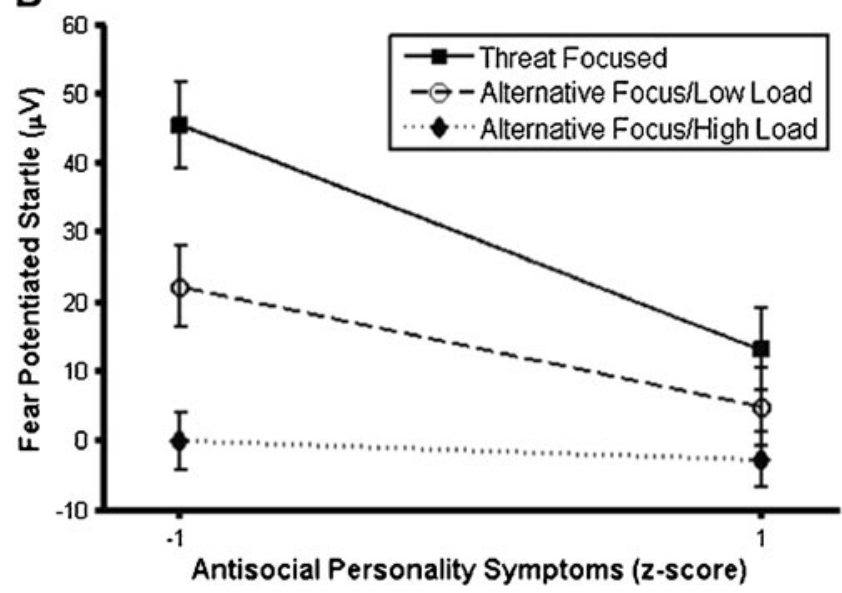

Fig. 2 Fear-potentiated startle as a function of PCL-R psychopathy $( \pm 1 S D$ from the mean; panel A), APD symptoms $( \pm 1 S D$ from the mean; panel B), and condition. Error bars represent the standard errors for the point estimates

when evaluating the effects of condition on psychopathy and APD (see below).

Unique effects of psychopathy total score and APD symptoms The main effect of psychopathy did not approach statistical significance $(p=.35)$, indicating that psychopathy total scores were not consistently related to P1 across conditions. However, there was a significant Psychopathy $\times$ Attentional Focus Contrast interaction, $F \times$ $(1,71)=4.37, p=.04, \eta_{p}^{2}=.058$, indicating that higher levels of psychopathy were associated with smaller P1 responses in the TF condition and larger $\mathrm{P} 1$ responses in the AF conditions. Owing to the symmetry of the interaction, neither simple effect was statistically significant (see Table 3).

For the unique effect of APD symptoms on the P1 response, neither the main effect $(p=.11)$ nor either interaction contrast was significant (all $p$ values $>.35$ ). 
Table 3 General linear model point estimates (and standard errors) for event-related potentials associated with no-threat and threat cues as a function of Psychopathy Checklist-Revised (PCL-R) psychopathy total score, antisocial personality disorder (APD), and task condition

Low PCL-R High PCL-R Low APD High APD

$\mathrm{P} 1$

Threat Focused

$\begin{array}{lllll}\text { No threat } & 1.78(.68) & 1.19(.68) & .57(.68) & 1.90(.68) \\ \text { Threat } & 2.76^{* \dagger}(.72) & .76^{* \dagger}(.72) & .79(.72) & 2.20(.72) \\ \text { Alternative Focus/Low Load } & & \\ \text { No threat } & 1.03(.62) & 1.56(.62) & .07^{*}(.62) & 1.85^{*}(.62) \\ \text { Threat } & 2.38^{*}(.81) & 1.63^{*}(.81) & .87(.81) & 2.52(.81) \\ \text { Alternative Focus/High Load } & & \\ \text { No threat } & 1.14(.67) & 1.62(.67) & .63(.67) & 1.72(.67) \\ \text { Threat } & 1.25(.80) & 1.67(.80) & .22(.80) & 2.02(.80) \\ \text { P3 } & & & \\ \text { Threat Focused } & & & \\ \text { No threat } & 11.92(1.21) & 10.96(1.21) & 11.04(1.21) & 11.83(1.21) \\ \text { Threat } & 14.60(1.30) & 11.87(1.30) & 11.68(1.30) & 14.80(1.30) \\ \text { Alternative Focus/Low Load } & & \\ \text { No threat } & 9.38(1.22) & 9.78(1.22) & 9.57(1.22) & 9.52(1.22) \\ \text { Threat } & 10.68(1.19) & 10.17(1.19) & 10.27(1.19) & 10.58(1.19) \\ \text { Alternative } & \text { Focus/High Load } & & \\ \text { No threat } & 7.89(1.04) & 9.68(1.04) & 8.74(1.04) & 8.83(1.04) \\ \text { Threat } & 7.78^{*}(.96) & 10.49^{*}(.96) & 9.56(.96) & 8.71(.96)\end{array}$

Point estimates are provided for low psychopathy and APD (1 standard deviation below sample mean) and high psychopathy and APD (1 standard deviation above sample mean). Flagged cells indicate significant $(p<.05)$ zero-order $\left({ }^{*}\right)$ and partial $\left(^{\dagger}\right)$ correlations involving PCL-R or APD scores. P1 was estimated at occipital sites (O1 and O2), and $\mathrm{P} 3$ was estimated at $\mathrm{Pz}$

Unique effects of psychopathy factor scores and APD symptoms The unique effect of PCL-R Factor 2 was significantly associated with the attentional focus contrast, $F \times$ $(1,70)=7.48, p=.01, \eta_{p}^{2}=.10$. Factor 2 was negatively associated with $\mathrm{P} 1$ responses in the TF condition but positively associated with $\mathrm{P} 1$ in the AF conditions, though none of the simple effects were significant. PCL-R Factor 1 was not associated with the $\mathrm{P} 1$ response (all $p$ values $>.35$ ). Also, the unique effects of APD were essentially equivalent to those found in the analysis using PCL-R total scores.

\section{Late attention (P3) analyses}

We found a significant main effect of cue type, $[F(1,73)=$ $\left.25.03, p .01, \eta_{p}^{2}=.26\right]$, indicating greater $\mathrm{P} 3$ responses on threat than on no-threat trials. There was also a significant effect of condition, $\left[F(1,73)=31.33, p .01, \eta_{p}^{2}=.30\right]$. The $\mathrm{P} 3$ response was significantly larger in the TF condition than in the AF conditions (attentional focus contrast; $p<.01$ ) and significantly larger in the AF/LL than in the AF/HL condition (cognitive load contrast; $p=.03$ ).

Unique effects of psychopathy total score and APD symptoms The main effect of psychopathy, controlling for APD, did not approach statistical significance $(p=.95)$, suggesting that psychopathy total scores were not consistently related to $\mathrm{P} 3$ across conditions.

However, a significant interaction did emerge between psychopathy and the attentional focus contrast, $F(1,71)=$ $8.42, p=.01, \eta_{p}^{2}=.106$, with individuals high on psychopathy displaying weaker $\mathrm{P} 3$ responses in the TF condition and stronger P3 responses in the AF conditions (see Table 3). There was no significant interaction involving psychopathy and cue type, and none of the simple effects within conditions were significant.

The main effect of APD, controlling for psychopathy, also failed to approach statistical significance $(p=.74)$, indicating that APD symptoms were not related to P3 response across conditions. However, the APD $\times$ Attentional Focus Contrast $\times$ Cue Type interaction was significant, $F(1,71)=4.38, p=.04, \eta_{p}^{2}=.058$. Individuals with high APD symptoms displayed a greater P3 to threat cues than to no-threat cues in the TF condition, whereas the $\mathrm{P} 3$ response in the AF conditions was greater for no-threat than for threat cues (see Table 3). None of the simple effects within conditions were significant.

Unique effects of psychopathy factor scores and APD symptoms This analysis revealed a significant PCL-R Factor $1 \times$ Attentional Focus Contrast interaction, $F \times$ $(1,70)=5.00, p=.03, \eta_{p}^{2}=.067$. Regardless of cue type, PCL-R Factor 1 scores were slightly negatively associated with $\mathrm{P} 3$ in the TF condition, but positively associated with P3 in the AF conditions. None of the other main effects or interactions involving Factor 2 attained statistical significance (all $p$ values $>.39$ ). The effects of APD were essentially equivalent to those reported above (i.e., in the analyses using PCL-R total scores).

\section{Discussion}

The purpose of this study was to examine and differentiate between the cognitive and affective processes associated with psychopathy and APD symptoms in female offenders. Using an instructed fear-conditioning paradigm that manipulated attentional focus and cognitive load, we examined the unique effects of psychopathy and APD symptoms on psychophysiological markers of cognitive and affective processes. As 
expected, psychopathy and APD were associated with distinct cognitive and affective patterns.

\section{Psychopathy}

Clarifying the dysfunctional cognitive and affective processes that potentiate antisocial behavior in psychopathy is crucial for advancing understanding and treatment of this disorder in female offenders. The present results (i.e., no main effect of psychopathy across conditions) are inconsistent with the existence of an absolute fear deficit in psychopathic female offenders. There was, however, support for the proposal that attentional focus moderates fear processing in psychopathic female offenders. According to Newman and colleagues, psychopathic offenders are characterized by abnormalities in early selective attention that facilitate processing of primary, goal-relevant information at the expense of peripheral information (Newman \& Baskin-Sommers, 2011). In the present study, female psychopathic offenders displayed stronger startle responses when instructed to focus on threat-relevant stimuli (i.e., the TF condition) than when they were required to focus on threat-irrelevant information (i.e., the AF conditions). These results are consistent with the notion that psychopathic women are affected by an attention abnormality that results in the prioritization of goalrelevant versus goal-irrelevant information. Furthermore, for these psychopathic females this prioritization may result in exaggerated fear reactivity when they focus directly on threatening information.

Examination of the ERP data further aids in the interpretation of this attentional effect. The P1 response provides an index of early selective attention and may be interpreted as the strength of the attention response to goal-relevant information (Hillyard \& Anllo-Vento, 1998). The P3 is an index of processing at a later stage and more often is associated with capacity-limited processes (e.g., categorization of information, working memory, or response selection; Kok, 2001). Psychopathic women displayed relatively larger P1 and P3 responses when they were instructed to focus on threatirrelevant information (i.e., AF conditions), but displayed smaller P1 and P3 responses under conditions involving a direct focus on threat (i.e., the TF condition). One interpretation of these findings is that psychopathic offenders are especially adept at screening out distracting threat-related information under conditions that allow them to anticipate and allocate attention to the goal-relevant aspects of a stimulus prior to its presentation (i.e., AF conditions). Conversely, when attending to threat-relevant cues is required for performing a task (i.e., TF condition), the threat information hampers their capacity for goal-relevant response selection. The suggestion that goal-relevant fear (i.e., threat) information interferes with response selection in psychopathic female offenders under threat-focused conditions is also consistent with their increased fear reactivity (i.e., FPS) and reduced P3 response under such conditions.

Some differences notwithstanding, these findings are consistent with those from male psychopathic offender populations. In a male offender sample, psychopathy scores were significantly and inversely related to FPS under alternativefocus conditions and positively, albeit nonsignificantly, related to FPS in threat-focused conditions (Newman et al., 2010). Moreover, examination of an early ERP indicated that, like female psychopathic offenders, male psychopathic offenders displayed a larger early ERP under alternative-focus than under threat-focused conditions (Baskin-Sommers, Curtin, Li, \& Newman, 2011). Thus, both male and female psychopathic offenders appear to display an early attention bias that prioritizes goal-relevant information. However, there is a slight difference in the pattern of fear reactivity displayed in each sample: Psychopathic female offenders display significant greater fear responses in the threat-focused condition, whereas psychopathic male offenders display significant weaker fear responses in the alternative-focus conditions.

Although attention moderates the fear responses of psychopathic males and females in a similar way, it is possible that differences in the patterns of these effects across conditions reflect important gender-specific tendencies in emotion processing. Numerous studies have found gender differences in perceiving, interpreting, and reacting to emotion cues (Bradley, Cuthbert, \& Lang, 1999; Galen \& Underwood 1997; Goos \& Silverman, 2002; McClure, 2000; McClure et al., 2004; Vitale et al., 2011). For instance, as compared to men, women tend to show greater increases in skin conductance and stronger startle potentiation to threatening scenes (Bradley et al., 1999; McManis, Bradley, Berg, Cuthbert, \& Lang, 2001). Thus, the results of the present study may reflect the combination of a gender-related sensitivity to fear and a psychopathy-related abnormality in selective attention that decreases responsivity to threat cues that are peripheral to one's goal-directed focus of attention. More specifically, the inflated fear responses of psychopathic women may reflect their tendency to overallocate attention to primary, goal-relevant stimuli as well as a gender-related bias toward processing emotion information. However, when faced with competing demands to prioritize goal-relevant stimuli, psychopathic women may be unusually adept at screening out peripheral threat stimuli in order to remain focused on the goal-relevant stimuli (i.e., P1 and $\mathrm{P} 3$ effects). Owing to the tentative nature of these proposals, additional research will be needed to evaluate the role that gender-specific biases and attentional biases play in the disinhibited behavior of psychopathic female offenders.

\section{Psychopathy factors}

Another important factor that may contribute to the complexity of identifying and differentiating the psychobiological 
underpinnings of psychopathy and APD relates to the potential multidimensional nature of psychopathy. Factor analyses of multiple measures of psychopathy have revealed a reliable two-factor structure. The first factor (Factor 1) relates to the affective and interpersonal symptoms of psychopathy, whereas the second factor (Factor 2) relates to its impulsive and antisocial symptoms (Hare \& Neumann, 2009). In general, Factor 2 is highly predictive of antisocial conduct (i.e., APD), whereas Factor 1 tends to distinguish psychopathy from APD and other externalizing disorders (Verona \& Vitale, 2006; Warren et al., 2003). As such, it was expected that the results for Factor 1 would correspond most closely with the findings for psychopathy total scores and that the Factor 2 findings would correspond to the APD effects. However, the observed associations between the factors and diagnostic syndromes were not consistent with these straightforward expectations.

Contrary to the results for psychopathy total score, women with high Factor 1 scores showed no indication of differences in fear reactivity (i.e., FPS) or early selective attention (i.e., P1). On the other hand, resembling the results for high psychopathy scores, high scores on Factor 1 were associated with abnormalities in capacity-limited processes (i.e., P3). More specifically, high Factor 1 scorers displayed larger $\mathrm{P} 3$ responses in the $\mathrm{AF}$ conditions than in the TF condition. The latter finding may indicate that women high on Factor 1 traits utilize capacitylimited processes under conditions that require focusing on a goal (i.e., categorizing a letter) in the face of distraction (i.e., threat information) more than do women low on Factor 1 traits.

Surprisingly, the results from the fear reactivity and early selective attention measures revealed that women with high Factor 2 scores were more similar to the psychopathic women. Factor 2 was significantly and positively associated with FPS in the TF condition and negatively associated with FPS in the AF conditions. Additionally, women high on Factor 2 displayed larger $\mathrm{P} 1$ responses in the AF conditions than in the TF condition. Overall, it appears that, in women, Factor 2 and the unitary measure of psychopathy (i.e., total scores) share a psychobiological signature (i.e., display similar early attention and affective responses). Of note, across measures, none of the effects for Factor 2 resembled the APD effects.

Though a number of studies have replicated the PCL-R two-factor structure in women (see Verona \& Vitale, 2006), it is important to note that a few studies have failed to replicate this structure (Salekin et al., 1997; see Rogstad \& Rogers, 2008, for a review). Additionally, there is some evidence that the external correlates of the factors in women do not always conform to expectations. For instance, Hazelwood and Rogers (as submitted) reported that Factor 1 was more strongly related to APD than was Factor 2 (see also Rutherford, Alterman, Cacciola, \& McKay, 1998; cf. Warren et al., 2003). In the present study, the results for APD and Factor 2 did not resemble one another. However, in light of our unexpected results and other inconsistencies in this domain, we believe that it is premature to draw conclusions regarding the relationships between the PCL-R factors and psychopathy or APD in women on the basis of the present evidence.

Antisocial personality disorder symptoms

Contrary to expectations, we found no evidence that women with more APD symptoms were hyperreactive to threat stimuli. In fact, women with elevated APD symptoms showed significantly less FPS across conditions, and this effect was strongest in the TF condition.

In line with these findings, women with high APD symptoms displayed greater P3 responses to threat than to nothreat stimuli in the $\mathrm{TF}$ condition relative to the AF conditions. The greater $\mathrm{P} 3$ response suggests that, under conditions with minimal cognitive demands (e.g., the TF condition), they were better able to identify the correct response, particularly to threat cues. Conversely, women with high APD symptoms displayed smaller P3 responses in the AF conditions, suggesting that they were less able to identify the correct responses under conditions that placed higher demands on capacity-limited processing resources. This pattern of findings is partially consistent with proposals linking APD to deficient executive functioning (i.e., cognitive control; working memory capacity), though it is contrary to the prediction that this deficit would yield affective hyperreactivity (i.e., the present study found deficient fear reactivity). While necessarily post hoc, one plausible interpretation of these findings is that women with elevated APD symptoms employ capacity-limited resources to reduce fear responding in the TF condition, resulting in larger P3 responses and significantly diminished FPS, but are less able to do so under conditions that deplete cognitive resources, resulting in greater fear-related distraction, smaller P3 responses, and nonsignificantly smaller FPS. Though speculative, this interpretation is consistent with expectations regarding the association between limited executive functioning and APD symptoms in female offenders.

To our knowledge, this study is the first to identify an association between APD and low fear responses in adult female offenders. However, using adolescents, Fairchild, Stobbe, van Goozen, Calder, and Goodyer (2010) recently reported that girls with conduct disorder displayed significantly weaker fear conditioning than did controls. Moreover, the presence of a fear deficit in females with APD is consistent with some studies that have found affective deficits in males with APD and aggressive behaviors (Gao, Raine, Venables, Dawson, \& Mednick, 2010; Raine, Lencz, Bihrle, LaCasse, \& Colletti, 2000).

In conjunction with previous research, the present findings suggest that females with APD symptoms display affective deficits similar to those of their male counterparts, and may attempt to use capacity-limited resources to reduce their 
reactivity to fear information. However, possibly related to weak executive functioning, they appear to be less able to do this when cognitive load increases. Overall, a clinically significant link between fearlessness and antisocial behavior in female offenders emerges that warrants further study.

\section{Limitations}

A potential limitation of this study concerns the high correlation between the total psychopathy and APD scores (see Table 1). On the one hand, the magnitude of this association highlights the importance of examining psychopathy and APD effects simultaneously, as advocated by Vaidyanathan et al. (2011). Without partialing the effects from each other (i.e., analyzing only zero-order correlations), it would be difficult to determine whether the zero-order correlates of psychopathy and APD were confounded by the other variable. On the other hand, when interpreting the present results, it is crucial to consider that our analyses examining their simultaneous effects served to highlight the unique variance associated with each variable rather than psychopathy or APD per se (i.e., combinations of shared and unique variance). As clarified by Lynam, Hoyle, and Newman (2006), characterizing the unique variance associated with highly correlated variables is often problematic. Nevertheless, to the extent that the zero-order and partialed effects converge, one may have greater confidence that the unique effects pertain to the overall construct (e.g., psychopathy or APD regardless of partialing). In this regard, it is worth noting that the zero-order correlations for psychopathy and APD resemble those obtained in the primary, partialed analyses.

Another potential limitation pertains to our interpretation of the ERP data in this study. Accurate interpretation of ERPs is challenging under any circumstances, but is especially difficult in the absence of a substantial research literature involving the experimental paradigm. To date, only one publication has examined ERPs within the context of this paradigm (Baskin-Sommers et al., 2011). Thus, our interpretation of the present findings is necessarily tentative. Nevertheless, we believe that the ERP data make a valuable contribution to the present study and will become increasingly important as the ERP literature on female offenders continues to develop.

\section{Summary and conclusions}

The purpose of this study was to address the dearth of laboratory evidence regarding dysfunctional cognitive and affective processes in psychopathy and APD. Importantly, we found that the same dysfunctional processes do not characterize psychopathy and APD. That is, while their behaviors and antisocial lifestyles may be similar, the cognitive and affective correlates of these two syndromes appear to be quite different.

To the extent that the disinhibited behavior of psychopathic female offenders is not attributable to an absolute deficit in fear processing, it is important to consider other potential mechanisms. As was clarified by MacCoon, Wallace, and Newman (2004), maintaining a context-appropriate balance of attention between top-down goals and bottom-up peripheral stimuli is crucial for self-regulation. In as much as psychopathic female offenders allocate attention to primary concerns and overlook peripheral cues that might otherwise moderate goal-directed behavior, their attention-related abnormality may leave them vulnerable to impulsive, poorly regulated action in situations involving motivationally significant cues that capture their attention (MacCoon et al., 2004).

Classic portrayals of psychopathy emphasize deficits in emotion processing (Cleckley, 1976), and especially fear processing (Lykken, 1995). On the one hand, the elevated FPS displayed by female psychopaths in the TF condition appears to depart from this classic characterization of psychopathy. On the other, this apparent sensitivity to threat cues is undetectable when it conflicts with their attainment of a proximal goal, as indicated by the P1, P3, and alternative-focus FPS effects found in this study. Thus, despite their apparent gender-related tendency to monitor emotion information, psychopathic women appear to possess a distinctive capacity to filter out threat-related information when it conflicts with a goal. Moreover, it is possible that this capacity may contribute significantly to their selfish remorseless exploitation of others.

In contrast to our findings for psychopathy, a fear deficit was clearly apparent in female offenders with high APD scores. Moreover, women with APD displayed deficits in capacity-related processing when cognitive demands were high, as indicated by the decreased $\mathrm{P} 3$ response in the AF versus the TF conditions. Of note, though, when there was little demand on cognitive processing, women with elevated APD symptoms were able to employ capacity-related resources to reduce reactivity to the threat information, as indicated by the $\mathrm{P} 3$ effect described above and the significant reduction in FPS in the TF condition.

As described by Lykken (1995), antisocial individuals may overlook threat information for multiple reasons, including an innate fear deficit, excessive approach motivation related to personally relevant goals (e.g., sex, drugs, or money), and failures of socialization associated with impoverished environments, gang membership, and defective parenting (see also Javdani, Sadeh, \& Verona, 2011). Increasing evidence is also linking adult antisocial behavior to early child abuse (Javdani et al., 2011; van Goozen, Snoek, Matthys, van Rossum, \& van Engeland, 2004). Although we did not directly investigate the source of their propensity to screen out threat-related information, one possibility is that the propensity to ignore 
threat cues in female offenders with high APD scores is linked to a more general failure of socialization. To the extent that one's environment is overly harsh and unpredictable during development, this may foster a cognitive bias that favors the tuning out of noncontingent aversive events (Evans, 1982). Regardless of the etiology, a stable tendency to ignore threat cues that inhibit antisocial behaviors in others is likely to increase a person's risk for diverse criminal behaviors (see Lykken, 1995).

The present findings begin to identify potential psychobiological mechanisms operating in psychopathy and APD and to highlight important syndrome-related differences in these mechanisms. The findings for psychopathy suggest that abnormalities in selective attention may play a crucial role in their cognitive and affective disinhibition. Alternatively, the findings for APD highlight deficiencies in fear processing and, potentially, deficits in executive functions that may hamper goal-directed behavior under conditions involving relative high cognitive load. Given the general dearth of laboratory evidence on female offenders, we regard the present findings as providing a tentative framework for future work on cognitive-affective processes in psychopathy and APD, rather than as definitive evidence. Much more work will be needed to understand the impacts of gender, specific psychobiological mechanisms, and psychopathology in female offenders.

Author note The first two authors contributed equally to this work, which was supported by NIMH Grant Nos. 5R01MH078980 and T32MH018931. We thank many at the Wisconsin Department of Corrections and the Robert E. Ellsworth Correctional Institution for making this research possible.

\section{References}

Baskin-Sommers, A. R., Curtin, J. J., Li, W., \& Newman, J. P. (2011). Psychopathy-related differences in selective attention are captured by an early event related potential. Personality Disorders: Theory, Research and Treatment. doi:10.1037/a0025593

Baskin-Sommers, A. R., \& Newman, J. P. (in press). Differentiating the cognition-emotion interactions that characterize psychopathy versus externalizing disorders. In M. D. Robinson, E. Harmon-Jones, \& E. R. Watkins (Eds.), Cognition and emotion. New York, NY: Guilford Press.

Baskin-Sommers, A. R., Newman, J. P., Sathasivam, N., \& Curtin, J. J. (2011). Evaluating the generalizability of a fear deficit in psychopathic African American offenders. Journal of Abnormal Psychology, 120, 71-78. doi:10.1037/a0021225

Beauchaine, T. P., Klein, D. N., Crowell, S. E., Derbidge, C., \& Gatzke-Kopp, L. (2009). Multifinality in the development of personality disorders: A Biology $\times \mathrm{Sex} \times$ Environment interaction model of antisocial and borderline traits. Development and Psychopathology, 21, 735-770. doi:10.1017/S0954579409000418

Blair, R. J. R. (2003). Neurobiological basis of psychopathy. The British Journal of Psychiatry, 182, 5-7.

Bradley, M. M., Cuthbert, B. N., \& Lang, P. N. (1999). Affect and the startle reflex. In M. E. Dawson, A. M. Schell, \& A. H. Böhmelt
(Eds.), Startle modification: Implications for neuroscience, cognitive science, and clinical science (pp. 157-183). New York, NY: Cambridge University Press.

Cleckley, H. M. (1976). The mask of sanity (5th ed.). St. Louis, MO: Mosby (Original work published 1941).

Curtin, J. J. (2011). PhysBox: The Psychophysiology toolbox: A Matlab toolbox for reducing psychophysiological data within EEGLab. Retrieved from http://dionysus.psych.wisc.edu/ PhysBox.htm

Davis, M., Falls, W. A., Campeau, S., \& Kim, M. (1993). Fearpotentiated startle: A neural and pharmacological analysis. Behavioural Brain Research, 58, 175-198.

Delorme, A., \& Makeig, S. (2004). EEGLAB: An open source toolbox for analysis of single-trial EEG dynamics including independent component analysis. Journal of Neuroscience Methods, 134, 921. doi:10.1016/j.jneumeth.2003.10.009

Dolan, M., \& Völlm, B. (2009). Antisocial personality disorder and psychopathy in women: A literature review on the reliability and validity of assessment instruments. International Journal of Law and Psychiatry, 32, 2-9.

Evans, G. W. (Ed.). (1982). Environmental stress. New York, NY: Cambridge University Press.

Fairchild, G., Stobbe, Y., van Goozen, S. H. M., Calder, A. J., \& Goodyer, I. M. (2010). Facial expression recognition, fear conditioning, and startle modulation in female subjects with conduct disorder. Biological Psychiatry, 68, 272-279.

Fazel, S., \& Danesh, J. (2002). Serious mental disorder in 23,000 prisoners: A systematic review of 62 surveys. Lancet, 16, 545550 .

Forster, K. I., \& Forster, J. C. (2003). DMDX: A Windows display program with millisecond accuracy. Behavior Research Methods, Instruments, \& Computers, 35, 116-124. doi:10.3758/BF03195503

Galen, B. R., \& Underwood, M. K. (1997). A developmental investigation of social aggression among children. Developmental Psychology, 33, 589-600.

Gao, Y., Raine, A., Venables, P. H., Dawson, M. E., \& Mednick, S. A. (2010). Association of poor childhood fear conditioning and adult crime. The American Journal of Psychiatry, 167, 56-60. doi:10.1176/appi.ajp.2009.09040499

Glenn, A. L., \& Yang, Y. (2012). The potential role of the striatum in antisocial behavior and psychopathy. Biological Psychiatry. doi:10.1016/j.biopsych.2012.04.027

Goos, L. M., \& Silverman, I. (2002). Sex related factors in the perception of threatening facial expressions. Journal of Nonverbal Behavior, 26, 27-41.

Hare, R. D. (2003). Manual for the Hare Psychopathy ChecklistRevised (2nd ed.). Toronto, Canada: Multi-Health Systems.

Hare, R. D., Harpur, T. J., \& Hakstian, A. R. (1990). The Revised Psychopathy Checklist: Reliability and factor structure. Psychological Assessment, 2, 338-341.

Hare, R. D., \& Neumann, C. S. (2009). Psychopathy: Assessment and forensic implications. Canadian Journal of Psychiatry, 54, 791-802.

Hillyard, S. A., \& Anllo-Vento, L. (1998). Event-related brain potentials in the study of visual selective attention. Proceedings of the National Academy of Sciences, 95, 781-787.

Javdani, S., Sadeh, N., \& Verona, E. (2011). Expanding our lens: Female pathways to antisocial behavior in adolescence and adulthood. Clinical Psychology Review, 31, 1324-1348.

Kennealy, P. J., Hicks, B. M., \& Patrick, C. J. (2007). Validity of factors of the Psychopathy Checklist-Revised in female prisoners. Assessment, 14, 323-340.

Kok, A. (2001). On the utility of P3 amplitude as a measure of processing capacity. Psychophysiology, 38, 557-577.

Litt, L. C., Hien, D. A., \& Levin, D. (2003). Adult antisocial behavior and affect regulation among crack/cocaine-using women. Psychology of Women Quarterly, 27, 143-152. 
Lorenz, A. R., \& Newman, J. P. (2002). Deficient response modulation and emotion processing in low-anxious Caucasian psychopathic offenders: Results from a lexical decision task. Emotion, 2, 91104.

Loucks, A. D. (1995). Criminal behavior, violent behavior, and prison maladjustment in federal female offenders. Unpublished doctoral dissertation, Queen's University, Kingston, Ontario, Canada.

Louth, S. M., Hare, R. D., \& Linden, W. (1998). Psychopathy and alexithymia in female offenders. Canadian Journal of Behavioural Science, 30, 91-98.

Luck, S. J., Woodman, G. F., \& Vogel, E. K. (2000). Event-related potential studies of attention. Trends in Cognitive Sciences, 4, 432-440. doi:10.1016/S1364-6613(00)01545-X

Lykken, D. T. (1995). The antisocial personalities. Hillsdale, NJ: Erlbaum.

Lynam, D. R., Hoyle, R. H., \& Newman, J. P. (2006). The perils of partialling: Examples from aggression and psychopathy. Assessment, 13, 328-341.

MacCoon, D. G., Wallace, J. F., \& Newman, J. P. (2004). Selfregulation: Context-appropriate balanced attention. In R. F. Baumeister \& K. D. Vohs (Eds.), Handbook of self-regulation: Research, theory, and applications (pp. 422-444). New York, NY: Guilford Press.

McClure, E. B. (2000). A meta-analytic review of sex differences in facial expression processing and their development in infants, children, and adolescents. Psychological Bulletin, 126, 424-453. doi:10.1037/0033-2909.126.3.424

McClure, E. B., Monk, C. S., Nelson, E. E., Zarahn, E., Leibenluft, E., Bilder, R. M., . . Pine, D. S. (2004). A developmental examination of gender differences in brain engagement during evaluation of threat. Biological Psychiatry, 55, 1047-1055. doi:10.1016/ j.biopsych.2004.02.013

McManis, M. H., Bradley, M. M., Berg, W. K., Cuthbert, B. N., \& Lang, P. J. (2001). Emotional reactions in children: Verbal, physiological, and behavioral responses to affective pictures. Psychophysiology, 38, 222-231. doi:10.1017/S0048577201991140

Morgan, A. B., \& Lilienfeld, S. O. (2000). A meta-analytic review of the relation between antisocial behavior and neuropsychological measures of executive function. Clinical Psychology Review, 20, 113-136.

Mulder, R. T., Wells, J. E., \& Bushnell, J. A. (1994). Antisocial women. Journal of Personality Disorders, 8, 279-287.

Neary, A. (1990). DSM 3 and Psychopathy Checklist assessment of Antisocial Personality Disorder in Black and White female felons. In R. D. Hare (Ed.), Manual for the Hare Psychopathy Checklist-Revised (2nd ed.). Toronto, Canada: MultiHealth Systems.

Newman, J. P., \& Baskin-Sommers, A. R. (2011). Early selective attention abnormalities in psychopathy: Implications for selfregulation. In M. I. Posner (Ed.), Cognitive neuroscience of attention (pp. 421-440). New York, NY: Guilford Press.

Newman, J. P., Curtin, J. J., Bertsch, J. D., \& Baskin-Sommers, A. R. (2010). Attention moderates the fearlessness of psychopathic offenders. Biological Psychiatry, 67, 66-70.

Ogilvie, J. M., Stewart, A. L., Chan, R. C. K., \& Shum, D. (2011). Neuropsychological measures of executive function and antisocial behavior: A meta-analysis. Criminology, 49, $1063-1107$.

Ogle, R. S., Maier-Katkin, D., \& Bernard, T. J. (1995). A theory of homicidal behavior among women. Criminology, 33, 173-193. doi:10.1111/j.1745-9125.1995.tb01175.x

Patrick, C. J. (2007). Getting to the heart of psychopathy. In H. Herve \& J. C. Yuille (Eds.), The psychopath: Theory, research, and social implications (pp. 207-252). Mahwah, NJ: Erlbaum.

Patrick, C. J., Hicks, B. M., Krueger, R. F., \& Lang, A. R. (2005). Relations between psychopathy facets and externalizing in a criminal offender sample. Journal of Personality Disorders, 19, 339-356. doi:10.1521/pedi.2005.19.4.339

Patterson, C. M., \& Newman, J. P. (1993). Reflectivity and learning from aversive events: Toward a psychological mechanism for the syndromes of disinhibition. Psychological Review, 100, 716-736. doi:10.1037/0033-295X.100.4.716

Pessoa, L., McKenna, M., Gutierrez, E., \& Ungerleider, L. G. (2002). Neural processing of emotional faces requires attention. Proceedings of the National Academy of Sciences, 99, 11458-11463. doi:10.1073/pnas. 172403899

Raine, A., Lencz, T., Bihrle, S., Lacasse, L., \& Colletti, P. (2000). Reduced prefrontal gray matter volume and reduced autonomic activity in antisocial personality disorder. Archives of General Psychiatry, 57, 119-127.

Rogstad, J. E., \& Rogers, R. (2008). Gender differences in the contributions of emotion to psychopathy and antisocial personality disorder. Clinical Psychology Review, 28, 1472 1484.

Rutherford, M. J., Alterman, A. I., Cacciola, J. S., \& McKay, J. R. (1998). Gender differences in the relationship of antisocial personality disorder criteria to Psychopathy Checklist-Revised scores. Journal of Personality Disorders, 12, 69-76.

Salekin, R. T., Rogers, R., \& Sewell, K. W. (1997). Construct validity of psychopathy in a female offender sample: A multitrait-multimethod evaluation. Journal of Abnormal Psychology, 106, 576585. doi:10.1037/0021-843X.106.4.576

Sargeant, M. N., Daughters, S. B., Curtin, J. J., Schuster, R., \& Lejuez, C. W. (2011). Unique roles of antisocial personality disorder and psychopathic traits in distress tolerance. Journal of Abnormal Psychology, 120, 987-992.

Singh, A. L., \& Waldman, I. D. (2010). The etiology of associations between negative emotionality and externalizing disorders. Journal of Abnormal Psychology, 119, 376-388.

Strachan, C. E. (1995). The assessment of psychopathy in female offenders. Dissertation Abstracts International: Section B. Sciences and Engineering, 55, 3634.

Sturek, J., Loper, A., \& Warren, J. (2008). Psychopathy in female inmates: The SCID-II Personality Questionnaire and the PCL-R. Psychological Services, 5, 309-319.

Sutton, S. K., Vitale, J. E., \& Newman, J. P. (2002). Emotion among females with psychopathy during picture perception. Journal of Abnormal Psychology, 111, 610-619.

Sylvers, P., Brennan, P. A., Lilienfeld, S. O., \& Alden, S. A. (2010) Gender differences in autonomic indicators of antisocial personality disorder features. Personality Disorders: Theory, Research, and Treatment, 1, 87-96.

Vaidyanathan, U., Hall, J. R., Patrick, C. J., \& Bernat, E. M. (2011). Clarifying the role of defensive reactivity deficits in psychopathy and antisocial personality using startle reflex methodology. Journal of Abnormal Psychology, 120, 253258. doi: $10.1037 / \mathrm{a} 0021224$

van Goozen, S. H. M., Snoek, H., Matthys, W., van Rossum, I., \& van Engeland, H. (2004). Evidence of fearlessness in behaviourally disordered children: A study on startle reflex modulation. Journal of Child Psychology and Psychiatry, 45, 884-892.

Verona, E., \& Vitale, J. E. (2006). Psychopathy in women: Assessment, manifestations, and etiology. In C. J. Patrick (Ed.), Handbook of psychopathy (pp. 415-436). New York, NY: Guilford Press.

Vitale, J. E., Brinkley, C. A., Hiatt, K. D., \& Newman, J. P. (2007). Abnormal selective attention in psychopathic female offenders. Neuropsychology, 21, 301-312. doi:10.1037/08944105.21.3.301

Vitale, J. E., MacCoon, G. D., \& Newman, J. P. (2011). Emotion facilitation and passive avoidance learning in psychopathic female offenders. Criminal Justice and Behavior, 38, 641-658. 
Vitale, J. E., \& Newman, J. P. (2001). Response perseveration in psychopathic females. Journal of Abnormal Psychology, 110, 644-647.

Vitale, J. E., Smith, S. S., Brinkley, C. A., \& Newman, J. P. (2002). The reliability and validity of the Psychopathy Checklist-Revised in a sample of female offenders. Criminal Justice and Behavior, 29, 202-231. doi:10.1177/0093854802029002005

Warren, J. I., Burnette, M. L., South, S. C., Chauhan, P., Bale, R., Friend, R., \& Van Patten, I. (2003). Psychopathy in women: Structural modeling and comorbidity. International Journal of Law and Psychiatry, 26, 223-242.
Warren, J. I., \& South, S. C. (2006). Comparing the constructs of antisocial personality disorder and psychopathy in a sample of incarcerated women. Behavioral Sciences \& the Law, 24, 1-20.

Zachary, R. A. (1986). Shipley institute of living scale: Revised manual. Los Angeles, CA: Western Psychological Service.

Zahn-Waxler, C., Shirtcliff, E. A., \& Marceau, K. (2008). Disorders of childhood and adolescence: Gender and psychopathology. Annual Review of Clinical Psychology, 4, 275-303.

Zlotnick, C. (1999). Antisocial Personality Disorder, affect dysregulation, and childhood abuse among incarcerated women. Journal of Personality Disorders, 13, 90-96. 